\title{
MicroRNA-505 suppresses gastric cancer cell proliferation and invasion by directly targeting Polo-like kinase-I
}

This article was published in the following Dove Medical Press journal: OncoTargets and Therapy

\author{
Sheng-Chun Dang' \\ Fei Wang' \\ Xiao-Bao Qian' \\ Malik Abdul' \\ Qais-Ahmad Naseer' \\ Wei Jin ${ }^{2}$ \\ Rong $\mathrm{Hu}^{3}$ \\ Qian $\mathrm{Gu}^{3}$ \\ Min $\mathrm{Gu}^{4}$ \\ 'Department of General Surgery, \\ Affiliated Hospital of Jiangsu \\ University, Zhenjiang, Jiangsu Province \\ 2I200I, People's Republic of China; \\ ${ }^{2}$ Department of Obstetrics and \\ Gynecology, The Changshu No. 2 \\ People's Hospital, Changshu, Jiangsu \\ Province 215500, People's Republic \\ of China; ${ }^{3}$ Department of Geriatrics, \\ Zhenjiang First People's Hospital, \\ Jiangsu Province 2I200I, People's \\ Republic of China; ${ }^{4}$ Department of \\ Oncology, Zhenjiang Hospital of \\ Traditional Chinese and Western \\ Medicine, Zhenjiang, Jiangsu 2I200 I, \\ People's Republic of China
}

Correspondence: Min Gu Department of Oncology, Zhenjiang Hospital of Traditional Chinese and Western Medicine, No. 18 Tuanshan Road, Zhenjiang, Jiangsu 2I 200I,

People's Republic of China

Tel/fax +86 5I I 88820988

Email dangscjda@I63.com
Purpose: The expression of microRNA-505 (miR-505) has been investigated in various cancers; however, its effect and mechanism in relation to gastric cancer (GC) are yet to be determined. Thus, the current evaluation aimed to examine the expression and potential role of miR-505 in GC. Materials and methods: Quantitative real-time PCR was carried out to analyze miR-505 expression in GC cells and tissues. We observed that miR-505 is differentially expressed in GC cells following transfection of its mimics or inhibitors. Changes in cell invasion, cell proliferation, and epithelial-mesenchymal transition markers were measured.

Results: These findings indicated that miR-505 expression is downregulated in both GC cell lines and GC tissues. In addition, knockdown miR-505 induced the invasion and proliferation of GC cells. Transfection of miR-505 mimics led to an elevation in N-cadherin expression but a decrease in E-cadherin expression. Furthermore, we have shown that miR-505 binds to the 3'-UTR region of Polo-like kinase-1.

Conclusion: Our results indicated that miR-505 suppresses GC cell proliferation and invasion; it may be a valuable candidate gene for seeking therapy strategy for GC.

Keywords: MicroRNA-505, EMT, polo-like kinase-1, gastric cancer

\section{Introduction}

Gastric cancer (GC) is a commonly occurring cancer. GC affects a large number of people around the world. ${ }^{1}$ For most GC patients, extensive invasion and lymphatic metastasis may have already occurred when diagnosed at a late stage., ${ }^{2,3}$ Therefore, investigation of useful diagnostic biomarkers for early GC diagnosis and to comprehend the GC mechanism is critical.

MicroRNAs (miRNAs) are small noncoding RNAs with regulatory functions. As reported recently, miRNAs play vital roles during the prognosis of GC, which includes cell proliferation, apoptosis, invasion, and so on. ${ }^{4-6}$ miRNAs generally interact with the $3^{\prime}$-UTR of target mRNAs, interfering with their translation or promoting degradation of the mRNAs. ${ }^{7,8}$ Recent investigations have shown that in endometrial cancer, ${ }^{9}$ osteosarcoma, ${ }^{10}$ hepatocellular carcinoma, ${ }^{11}$ and GC, ${ }^{12}$ microRNA-505 (miR-505) acts as a tumor suppressor. However, the latent mechanism of miR-505 in GC is still elusive, and thus the goal of the current evaluation was to elucidate the part miR-505 plays in GC.

\section{Materials and methods}

\section{Study participants and tissue collection}

Ten pairs of GC tissues and nearby healthy tissues of GC patients of the Affiliated Hospital of Jiang Su University treated from March 2015 and April 2017 were used 
in this study. The subjects did not receive chemotherapy or additional treatments before gastrectomy. Upon collection, the tissues were quickly flash-frozen in liquid nitrogen until they were evaluated and the experiment was conducted in accordance with the Declaration of Helsinki. Each one of the subjects provided informed written consent to take part in this evaluation. The Research Ethics Committee of the Affiliated Hospital of Jiang Su University (Zhen Jiang, People's Republic of China) approved this study.

\section{Cell culture and transfection}

The GES-1 cell line, a healthy gastric epithelial cell line, and four GC cell lines, which included SGC-7901, BGC823, MGC-803, and MKN45, were acquired from the Type Culture Collection of the Chinese Academy of Sciences (Shanghai, People's Republic of China). The cells were cultured in RPMI-1640 medium (Hyclone, Logan, MA, USA) supplemented with 10\% FBS (Gibco, Carlsbad, CA, USA) at $37^{\circ} \mathrm{C}$ and in a humidified $5 \% \mathrm{CO}_{2}$ atmosphere.

The miR-505 mimics and inhibitors as well as negative controls were both acquired from Biomics Biotechnologies Co., Ltd. (Guangdong, People's Republic of China). Lipofectamine $^{\mathrm{TM}} 3000$ reagent (Invitrogen, Carlsbad, CA, USA) was used for miRNA or siRNA transfection. miR-505 and target mRNA expression were assessed using quantitative real-time PCR (qRT-PCR) and Western blotting.

\section{Cell proliferation assay}

Cell proliferation was evaluated with the MTT proliferation assay following the manufacturer's instructions. ${ }^{10}$

\section{Cell invasion assays}

Cell invasion was determined by Transwell chamber assays in which the chambers were coated with Matrigel (BD Biosciences, San Jose, CA, USA). The MGC-803 and MKN45 cells were transfected with the miR-505 mimics, anti-miR-505, or negative controls (miR-NC) for 24 hours. Following transfection, the cells were gathered and then resuspended in serum-free RPMI-1640 medium, then 5,000 cells were added into each well of the Transwell chamber, followed by the addition of RPMI-1640 supplemented with 10\% serum into the lower chamber. Then, the cells were incubated for 24 hours with $5 \% \mathrm{CO}_{2}$ at $37^{\circ} \mathrm{C}$. Then, the cells were fixed in paraformaldehyde, dried, and then subjected to crystal violet staining. The transmembrane cells were then quantified using a microscope at a $200 \times$ magnification. Five fields were randomly taken and averaged. Three replicate wells were used in each experiment, and every experiment was performed in triplicate.

\section{Luciferase reporter assay}

TargetScan, miRBase, and Target PicTar software were used to predict the potential target genes for miR-505. A pmirGLO Dual-Luciferase miRNA target expression vector was employed for the 3'-UTR luciferase assays (Promega Corporation, Fitchburg, WI, USA). Following the protocol, the MGC-803 and MKN45 cells were cotransfected with the miR-505 mimics, anti-miR-505, or negative control as well as the pmirGLO Dual-Luciferase target expression vectors with Lipofectamine $^{\mathrm{TM}} 3000$ (Invitrogen) in the luciferase assay. Firefly as well as Renilla luciferase activities were evaluated using a fluorescence detector after an 18-hour transfection.

\section{qRT-PCR assay}

Guided by the company's directions, TRIzol (Invitrogen) was employed for total RNA extraction using GC and nearby healthy gastric tissues, as well as transfected MGC-803 or MKN45 cells. A microRNA qPCR detection kit (TaKaRa, Dalian, People's Republic of China) was employed to measure miR-505 expression levels, and an SYBR ${ }^{\circledR}$ PremixMix kit (Invitrogen) was in PCR amplification. By making use of the $2^{-\Delta \Delta C T}$ method, ${ }^{13}$ the miR505 and gene expression were normalized to U6 and $\beta$-actin expression. The primers for gene application were as follows: PLK1F 5'-GCAGCGTGCAGATCAACTTC-3' and PLK1-R 5'-AGGAGACTCAGGCGGTATGT-3'; N-cadherin-F 5'-TGGGAAATGGAAACTTGATGGC-3' and N-cadherin-R 5'-AGTTGCTAAACTTCACTGAAAGGA-3'; E-cadherin-F 5'-GCTGGACCGAGAGAGTTTCC-3' and E-cadherin-R 5'-CAAAATCCAAGCCCGTGGTG-3'; and $\beta$-actin-F $5^{\prime}$-GATGAGATTGGCATGGCTTT-3' and $\beta$-actin-R 5'-GTCACCTTCACCGTTCCAGT-3'.

\section{Western blotting assay}

The transfected cells were collected and then lysed with a RIPA lysis buffer (Beyotime, Shanghai, People's Republic of China) to collect total cellular proteins. Each sample was assayed for protein concentration by BCA assay kit (Biosharp, Anhui, People's Republic of China). Each protein sample was employed in $40 \mu \mathrm{g}$ of polyacrylamide gel electrophoresis using the earlier described concentrations, and then moved onto polyvinylidene difluoride membranes (Millipore, Billerica, MA, USA). Nonspecific binding was blocked using skim milk powder for 1 hour at room temperature, and then 
hybridized with anti-E-cadherin, anti-N-cadherin, and antiPLK1 (Abcam, Cambridge, MA, USA) at $4^{\circ} \mathrm{C}$ overnight. The next day, the tissues were incubated with anti-horseradish peroxidase-conjugated secondary antibodies (Santa Cruz Biotechnology Inc., Dallas, TX, USA) at room temperature for 2 hours. $\beta$-actin (Sigma-Aldrich Co., St Louis, MO, USA) was employed as an internal control. Protein expression was assessed using an X-ray film and enhanced chemiluminescence reagent detection system, and band densities were qualified using the ImageJ software (National Institutes of Health, Bethesda, MD, USA).

\section{Assessment of bromodeoxyuridine incorporation}

The treated or untreated cells that had been transfected with miR-505 mimics, anti-miR-505, or negative controls were rinsed with RPMI-1640 medium and then cultured in fresh medium with $10 \mu \mathrm{M}$ bromodeoxyuridine (BrdU; SigmaAldrich $\mathrm{Co}$ ) at room temperature for $\sim 1$ hour. After washing in cold PBS, the cells were then fixed in $70 \%$ ice-cold ethanol. Then, the cells were resuspended in $2 \mathrm{~mol} / \mathrm{L} \mathrm{HCl}$ at $37^{\circ} \mathrm{C}$ for 30 minutes, after which washing with PBS and hybridization using a mouse monoclonal anti-BrdU antibody (Abcam) at a dilution of 1:100 in PBST (PBS containing 0.1\% BSA and $0.2 \%$ Tween-20, $\mathrm{pH} 7.4$ ) at $4^{\circ} \mathrm{C}$ overnight took place. The next day, the cells were rinsed with PBST and then incubated with fluorescein isothiocyanate-conjugated rabbit anti-mouse immunoglobulin antibody (Jackson Immuno Research, Lancaster, PA, USA) at room temperature for approximately 2 hours. Then, the cells were stained with DAPI for around 10 minutes and then photographed.

\section{Statistical assay}

Data analysis was conducted with SPSS 19.0 (IBM, Chicago, IL, USA) and expressed as the mean \pm SD. Statistical significance was determined by ANOVA comparison among groups. Differences were deemed to be statistically significant at $P<0.05$.

\section{Results}

\section{MiR-505 is downregulated in GC tissues and GC cell lines}

qRT-PCR was employed to assess miR-505 expression in GC and nearby healthy gastric tissues, a normal gastric epithelial cell line, as well as different GC cell lines. Figure 1A reveals a decrease in miR-505 expression in GC tissues relative to nearby healthy tissues. Furthermore, miR-505 expression in GC cell lines were lower in contrast to the healthy gastric epithelial cell GES-1 (Figure 1B), as well as the SGC-7901 or BGC-823 cells. Because the expression of miR-505 was lower in MKN45 and MGC-803 cells, we selected and used these two cells in the subsequent experiments.
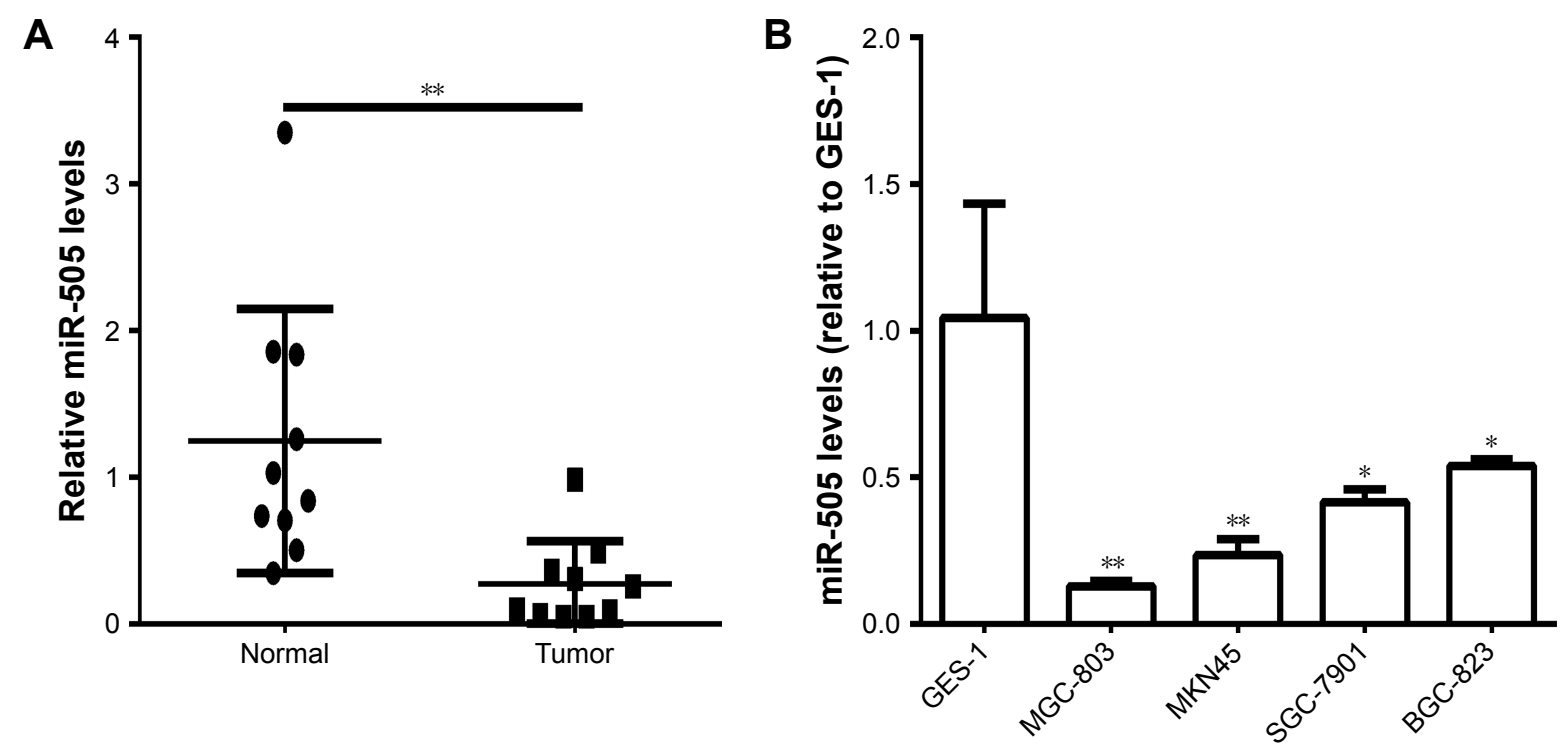

Figure I The expression of miR-505 is downregulated in gastric cancer tissues and gastric cancer cell lines.

Notes: (A) Expression of miR-505 in ten paired human gastric cancer ("Tumor") and nearby healthy gastric tissues ("Normal"). Expression of miR-505 in tumor decreased in contrast with the normal tissues. $* * P<0.01$. (B) Expression of miR-505 decreased in gastric cancer cell lines in contrast to healthy gastric cell GES-I. $* P<0.05$, $* * P<0.0 \mathrm{I}$. Data represent the mean \pm SD from three independent experiments.

Abbreviation: miR-505, microRNA-505. 


\section{MiR-505 disrupts GC proliferation and} invasion in vitro

Based on the results of the study, we transfected miR-505 mimic, anti-miR-505, or negative control miRNA (miR-NC) into MGC-803 and MKN45 cells separately. Figure 2A shows that compared to miR-NC and the control, miR-505 expression was significantly upregulated after transfection of the miR-505 mimic into the MGC-803 and MKN45 cells, whereas this was significantly downregulated after transfection with anti-miR-505. Compared to the miR-NC and the control, the miR-505 mimics inhibited GC cell prolifera- tion, whereas anti-miR-505 promoted GC cell proliferation (Figure 2B-E). In addition, compared to miR-NC and the control, GC cells transfected with the miR-505 mimics exhibited a lower rate of cell invasion, whereas GC cells transfected with anti-miR-505 showed a higher rate (Figure 3). Both results show that miR-505 can regulate GC carcinogenesis.

\section{MiR-505 inhibits epithelial-mesenchymal transition (EMT) in GC cell lines}

As reported, EMT indicates that many cancers begin to metastasize. ${ }^{14}$ Therefore, we want to explore whether
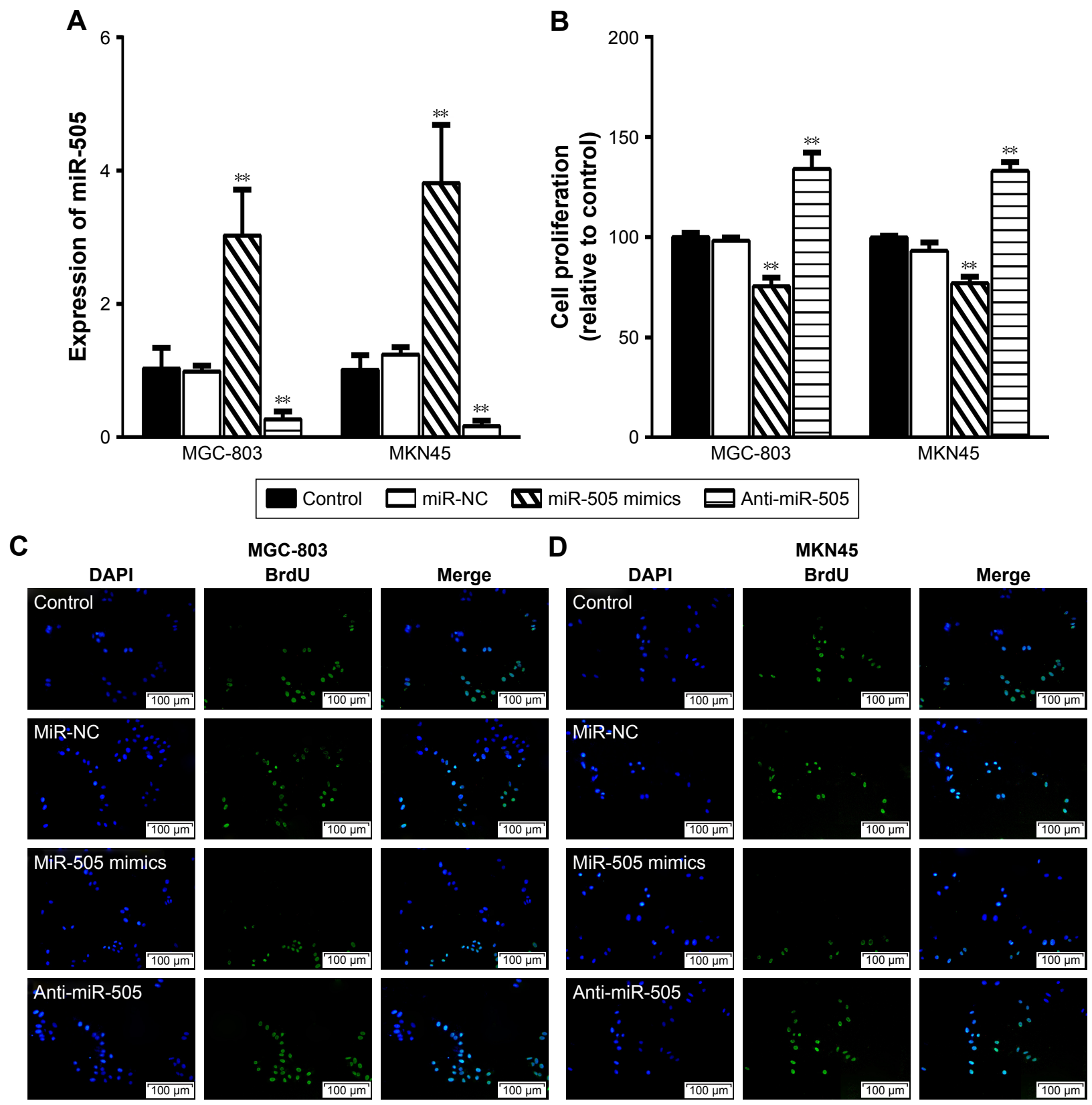

Figure 2 (Continued) 


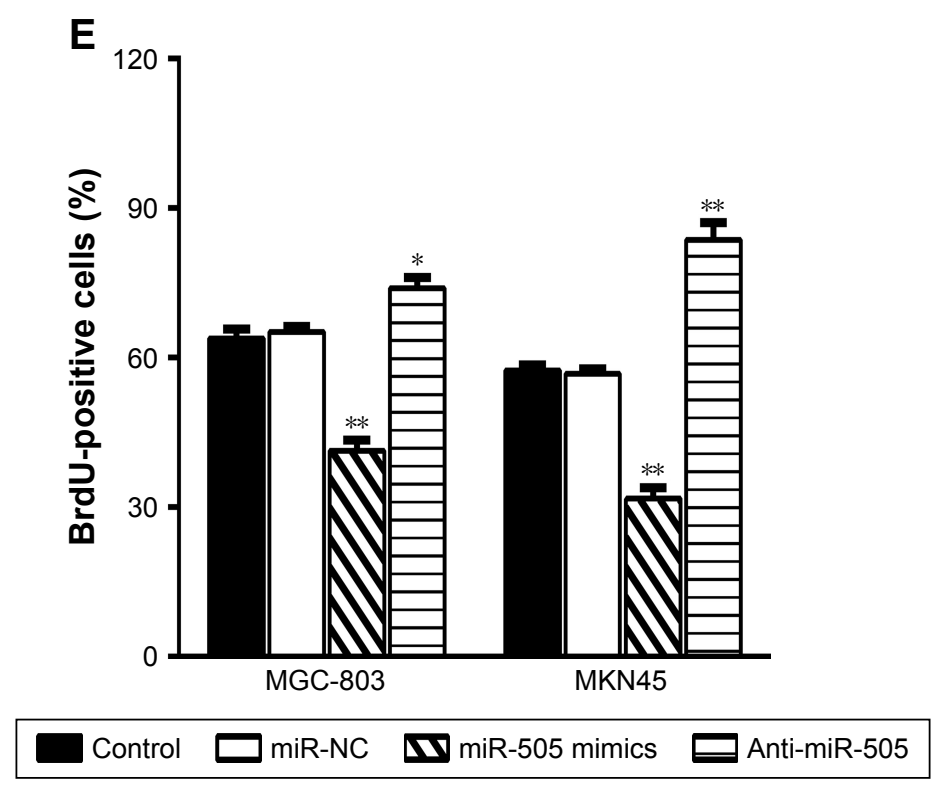

Figure 2 MiR-505 in vitro inhibited cell proliferation of gastric cancer cells.

Notes: (A) Expression of miR-505 in MGC-803 and MKN45 cells untransfected ("Control") or transfected with miR-505 mimic, anti-miR-505, and miR-NC. **P $<0.01$ vs Control. (B) Cell proliferation among MGC-803 and MKN45 cells untransfected ("Control”) or transfected with miR-505 mimics, anti-miR-505, or miR-NC. **P $<0.01$ vs Control. (C-E) Representative BrdU and DAPI immunofluorescence images in MGC-803 (C) and MKN45 (D) cells untransfected ("Control") or transfected with miR-505 mimics, anti-miR-505, or miR-NC, the BrdU-positive cells were calculated using Image software (E). $* P<0.05$ vs Control, $* * P<0.01$ vs Control. Data represent the mean \pm SD from three independent experiments. Magnification $\times 400$.

Abbreviations: BrdU, bromodeoxyuridine; miR-NC, negative control.

miR-505 causes EMT changes in GC. We transfected miR505 mimic, anti-miR-505, and miR-NC into MGC-803 and MKN45 cells and examined whether EMT-related proteins and their mRNA levels were altered. Figure 4 shows a rise in E-cadherin mRNA and protein expression and a decrease in N-cadherin mRNA and protein expression in both MGC803 and MKN45 cells after transfection with the miR-505 mimic. Moreover, E-cadherin expression decreased while $\mathrm{N}$-cadherin expression increased. These findings indicate that miR-505 suppresses EMT progression in the GC cell lines.

\section{MiR-505 directly controls PLKI expression in GC cell lines}

Three different databases, namely Target Scan, miRBase Target, and PicTar, were employed to predict the miR505 targets. We identified Polo-like kinase 1 (PLK1) as a direct target of miR-505. Earlier evaluations have revealed that PLK1 has a major part in regulating DNA synthesis, ${ }^{15}$ p53 transactivation, ${ }^{16}$ as well as tumor cell metastasis. ${ }^{17}$ So we hypothesized that miR-505 suppressed EMT progress probably through PLK1 molecules. To determine if miR-505 acts directly on PLK1, we performed a luciferase reporter assay. Figure 5A shows that miR-505 immediately binds to the $3^{\prime}$-UTR of PLK1. After transfecting the miR-505 mimetic into the MGC-803 and MKN45 cells, we observed a decrease in the luciferase activity of the 3 '-UTR of PLK1 in cells that were transfected with the miR-505 mimics relative to the negative controls. Furthermore, in both MGC-803 and MKN45 cells, PLK1 was reduced at the protein and its mRNA levels when transfected with miR-505 mimics compared to miR-NC. Moreover, PLK1 was elevated when transfected with anti-miR-505. These findings indicate that miR-505 directly disrupts PLK1 expression in GC cell lines.

\section{Discussion}

Advances in medicine have resulted in more effective and personalized treatment regimens for GC. However, the efficacy of these treatments significantly decreases in metastatic GC. The five-year overall survival rate for GC is currently $<20 \%$. ${ }^{18}$ Several miRNAs have been associated with GC. For instance, epigenetic regulation of miR-31, which targets HDAC2, inhibits GC cell proliferation, ${ }^{19}$ and miR-let-7a functions as a promoter in autophagy ${ }^{20}$ in GC. In addition, miR-143 has been shown to halt cell proliferation as well as prompt cell-cycle arrest at the G0/G1 phase in GC. ${ }^{4}$ Furthermore, miR-505 interferes with gankyrin expression by targeting the single-nucleotide polymorphism rs 111638916 in the $3^{\prime}$-UTR of the gankyrin gene, thus playing a protective role in GC pathogenesis. ${ }^{12}$ The current evaluation has shown that miR-505 is downregulated in GC tissues and cell lines 

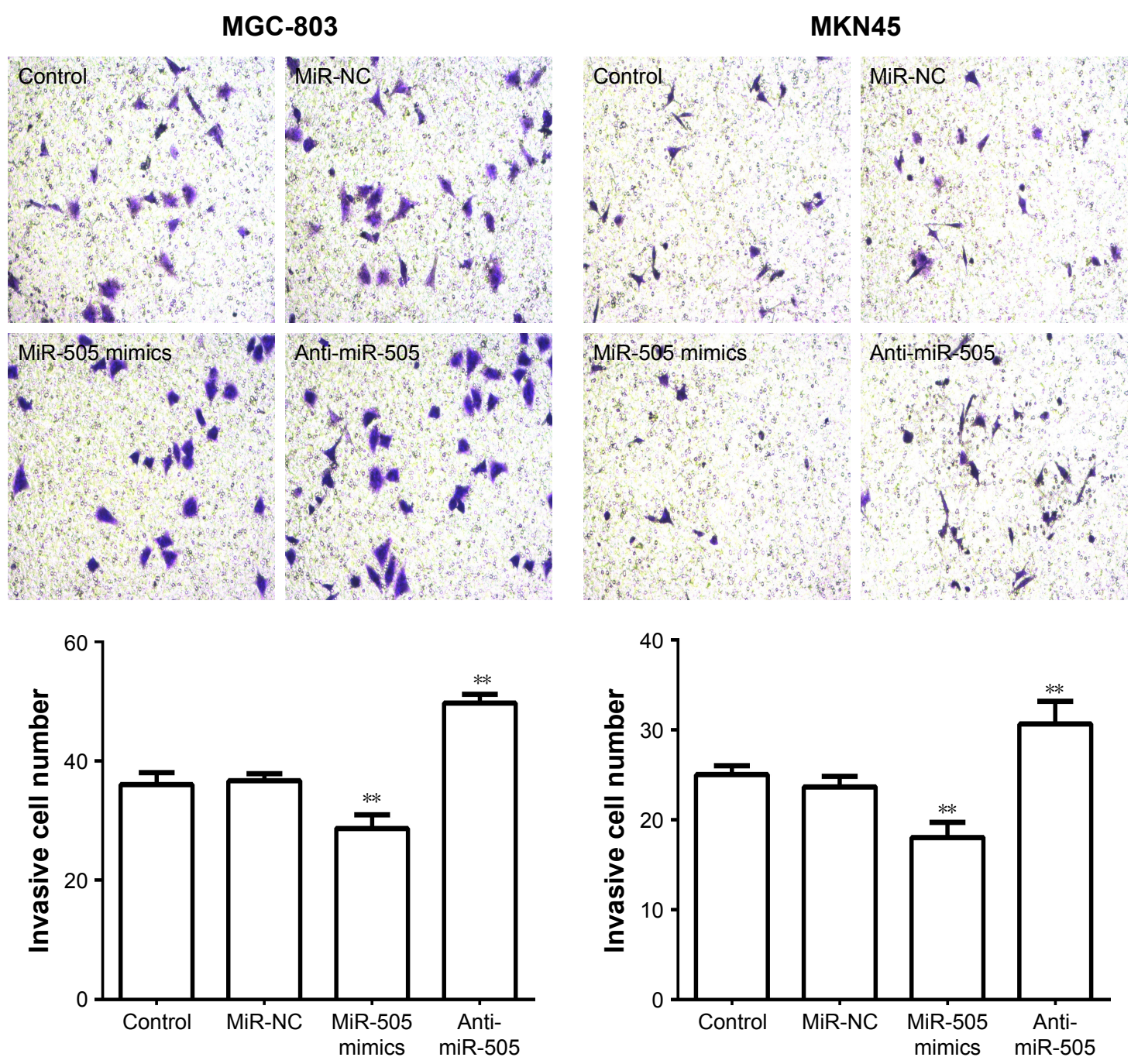

Figure 3 MiR-505 in vitro inhibited cell invasion of gastric cancer cells.

Notes: Typical cell invasion images of MGC-803 and MKN45 cells untransfected (“Control”) or transfected with miR-505 mimics, anti-miR-505, or miR-NC (200×). Taking five random fields for counting and drawing data into tables. $* * P<0.01$ vs Control. Data represent the mean $\pm S D$ from three independent experiments.

Abbreviation: miR-NC, negative control.

such as MGC-803 and MKN45. In addition, miR-505 upregulation suppresses MGC-803 and MKN45 cell proliferation and invasion. These discoveries suggest that miR-505 acts as a tumor suppressor in GC.

The EMT has a pivotal part in the metastasis of cancer, including GC. ${ }^{19-21}$ It can be found that miR-505 can inhibit EMT in vitro in human osteosarcoma cell MG63. ${ }^{10}$ Nonetheless, it was not clear that whether miR-505 could regulate EMT in GC cells. This study has shown that miR-505 upregulation in the MGC-803 and MKN45 cells results in an elevation in E-cadherin mRNA and protein expression, and a decline in N-cadherin mRNA and protein expression. The opposite results were achieved in MGC-803 and MKN45 cells when miR-505 was downregulated. These findings indicate that miR-505 suppresses EMT progression in GC cells.
We additionally examined how miR-505 disrupts GC cell proliferation and invasion. We determined that miR505 directly targets PLK1 by binding to its 3'-UTR. After upregulation of miR-505 in MGC-803 and MKN45 cells, the mRNA and protein levels of PLK1 decreased. Also, PLK1 increased when transfected with anti-miR-505. Based on the results, we can further infer that miR-505 is likely to suppress the prognosis of GC though PLK1. The previous studies have proven that PLK1 is a multifunctional and critical silk/ threonine kinase that plays a broad regulatory role in mitosis in cells. Its major function is to facilitate cells to enter mitosis as well as to regulate G2/M checkpoints. ${ }^{22}$ PLK1 upregulation promotes cellular proliferation and tumor transformation, whereas its downregulation induces tumor cell apoptosis and disrupt tumor cell growth. ${ }^{23,24}$ In addition, PLK1 drives gastric 
A

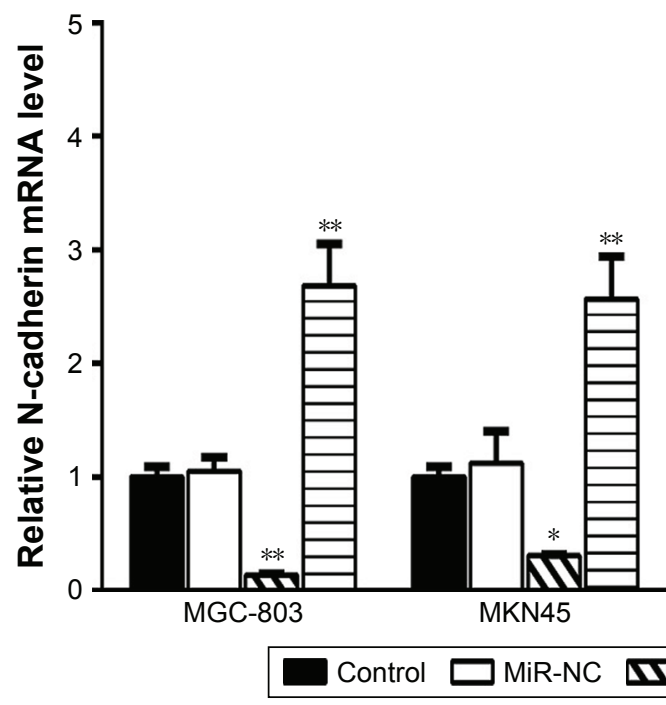

C

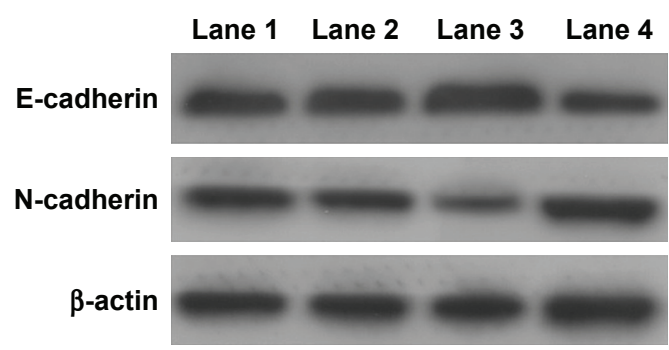

MGC-803 cell

Lane 1 Control

Lane 2 MiR-NC

Lane 3 MiR-505 mimics

Lane 4 Anti-miR-505

D

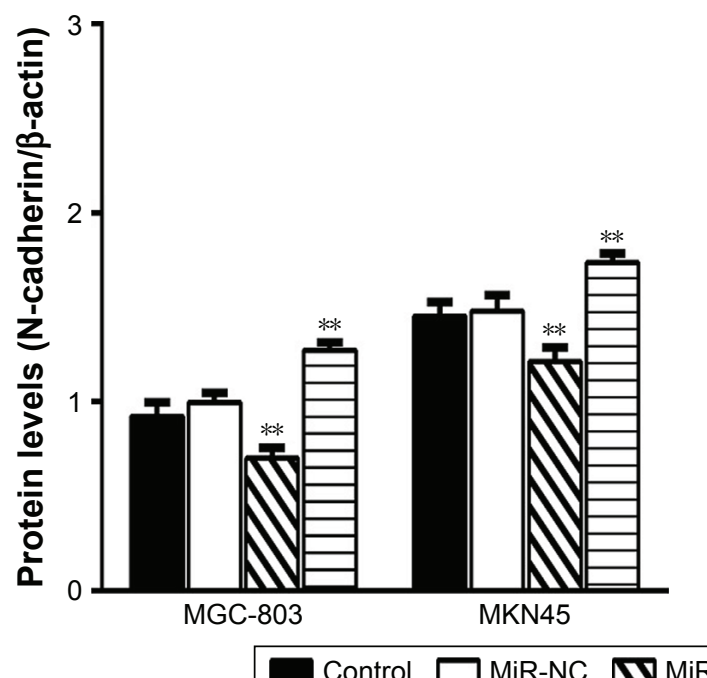

B

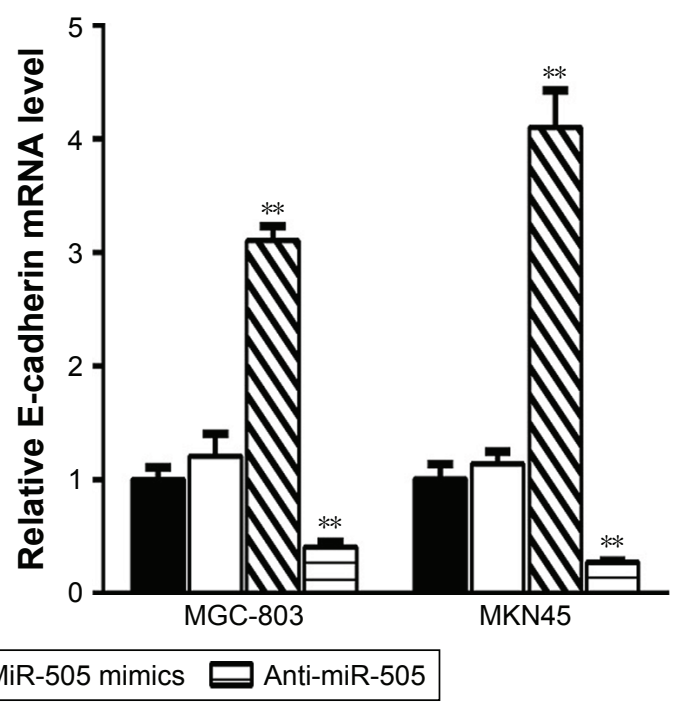


A Position of 52-73 of PLK1 3'-UTR 5'-gggc CCATACTGGTTGGCT CC C-3 hsa-miR-505 3'-uguaGUUAUG AAG GA CC |||||||| $\mid$
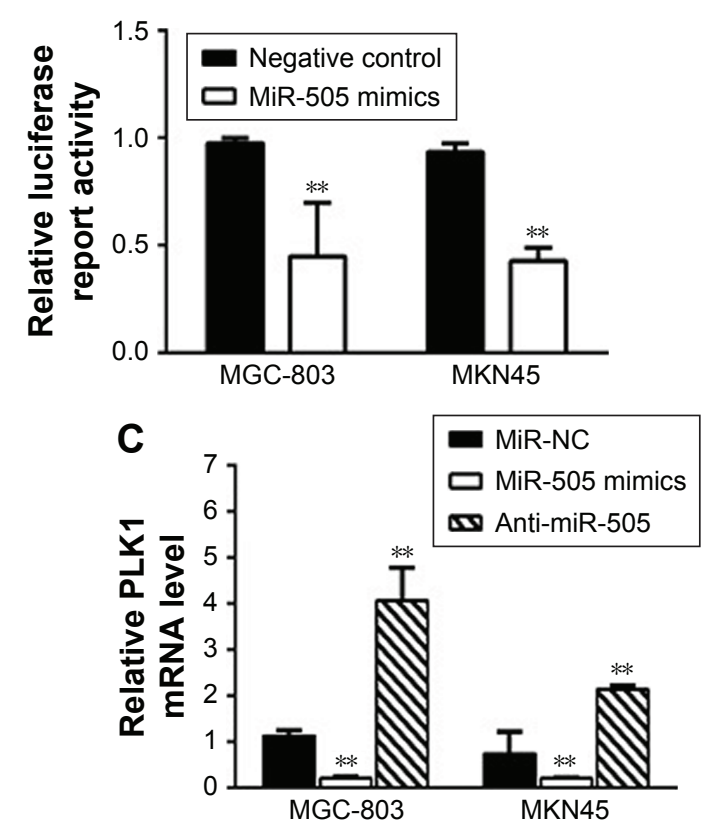

B
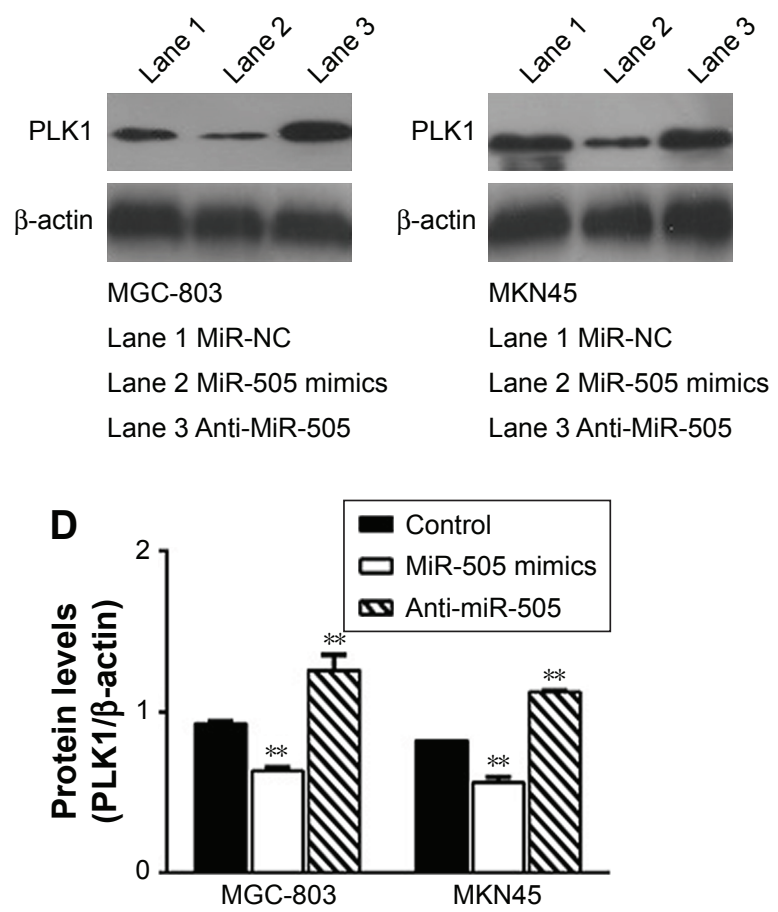

Figure 5 MiR-505 directly regulates PLKI expression in gastric cancer cells.

Notes: (A) Predicted seed sequences of miR-505 and 3'-UTR region of PLKI, also miR-505 imitates significantly lowered luciferase activity of 3 '-UTR of PLKI in contrast to the negative control. ${ }^{*} P<0.01$. (C) mRNA levels of PLKI in MGC-803 and MKN45 cells transfected with miR-505 mimics, anti-miR-505, or miR-NC. $* * P<0.01$ vs miR-NC. (B, D) Protein levels of PLKI in MGC-803 and MKN45 cells transfected with miR-505 mimics, anti-miR-505, or miR-NC. **P<0.0I vs miR-NC. Data represent the mean \pm SD from three independent experiments.

Abbreviations: miR-NC, negative control; PLKI, Polo-like kinase-I.

cell metastasis by mechanisms inducing EMT. ${ }^{25}$ Taken together, the discoveries of the current evaluation show that there is a great possibility that the mechanism of miR-505 involves the inhibition of GC cell proliferation and invasion by directly binding to PLK1. In addition, more markers of EMT will be further explored and animal experiments will be further carried out for verification and support.

\section{Conclusion}

The current evaluation has shown that miR-505 is downregulated in GC cell lines. In addition, it directly binds to PLK1 and inhibits EMT progression, thereby inhibiting MGC-803 and MKN45 cell proliferation and invasion in vitro. Further, miR-505 imparts an inhibitory effect on GC and thus may be potentially utilized as a new target for GC treatment.

\section{Acknowledgment}

The Six Talent Peaks Project in Jiangsu Province (No 2016-WSN-007), the Jiangsu 333 Project Foundation (No BRA2017560), the Zhenjiang Science and Technology Committee (No SH 2018061), the Changsu Science and
Technology Committee (No 20170016), and the Changshu Health Commisson Science and Technology Project (No CSWS 201801) supported this study.

\section{Disclosure}

The authors report no conflicts of interest in this work.

\section{References}

1. Jemal A, Bray F, Center MM, Ferlay J, Ward E, Forman D. Global cancer statistics. CA Cancer J Clin. 2011;61(2):69-90.

2. Dassen AE, Lemmens VE, van de Poll-Franse LV, et al. Trends in incidence, treatment and survival of gastric adenocarcinoma between 1990 and 2007: a population-based study in the Netherlands. Eur J Cancer. 2010;46(6):1101-1110.

3. Wu HH, Lin WC, Tsai KW. Advances in molecular biomarkers for gastric cancer: miRNAs as emerging novel cancer markers. Expert Rev Mol Med. 2014;16:e1.

4. Mao GP, Liu R, Qin YR. miR-143 inhibits cell proliferation of gastric cancer cells through targeting GATA6. Oncol Res. 2018;26(7): 1023-1029.

5. Zeng B, Shi W, Tan G. MiR-199a/b-3p inhibits gastric cancer cell proliferation via down-regulating PAK4/MEK/ERK signaling pathway. BMC Cancer. 2018;18(1):34.

6. Shen Y, Chen H, Gao L, et al. MiR-638 acts as a tumor suppressor gene in gastric cancer. Oncotarget. 2017;8(64):108170-108180. 
7. He L, Hannon GJ. MicroRNAs: small RNAs with a big role in gene regulation. Nat Rev Genet. 2004;5(7):522-531.

8. Fabian MR, Sonenberg N, Filipowicz W. Regulation of mRNA translation and stability by microRNAs. Annu Rev Biochem. 2010;79: 351-379.

9. Chen S, Sun KX, Liu BL, Zong ZH, Zhao Y. MicroRNA-505 functions as a tumor suppressor in endometrial cancer by targeting TGF- $\alpha$. Mol Cancer. 2016;15:11.

10. Liu YJ, Li W, Chang F, Liu JN, Lin JX, Chen DX. MicroRNA-505 is downregulated in human osteosarcoma and regulates cell proliferation, migration and invasion. Oncol Rep. 2018;39(2):491-500.

11. Lu L, Qiu C, Li D, Bai G, Liang J, Yang Q. MicroRNA-505 suppresses proliferation and invasion in hepatoma cells by directly targeting highmobility group box 1. Life Sci. 2016;157:12-18.

12. Liu Y, Xu J, Jiang M, Ni L, Chen Y, Ling Y. Association between functional PSMD10 Rs111638916 variant regulated by MiR-505 and gastric cancer risk in a Chinese population. Cell Physiol Biochem. 2015; 37(3):1010-1017.

13. Pfaffl MW. A new mathematical model for relative quantification in real-time RT-PCR. Nucleic Acids Res. 2001;29(9):e45.

14. Yang J, Weinberg RA. Epithelial-mesenchymal transition: at the crossroads of development and tumor metastasis. Dev Cell. 2008; 14(6):818-829.

15. Wakida T, Ikura M, Kuriya K, et al. The CDK-PLK1 axis targets the DNA damage checkpoint sensor protein RAD9 to promote cell proliferation and tolerance to genotoxic stress. Elife. 2017;6:e29953.

16. Smith L, Farzan R, Ali S, Buluwela L, Saurin AT, Meek DW. The responses of cancer cells to PLK1 inhibitors reveal a novel protective role for p53 in maintaining centrosome separation. Sci Rep. 2017;7(1):16115.
17. Fu Z, Wen D. The emerging role of polo-like kinase 1 in epithelialmesenchymal transition and tumor metastasis. Cancers (Basel). 2017;9(10):E131.

18. GASTRIC (Global Advanced/Adjuvant Stomach Tumor Research International Collaboration) Group, Oba K, Paoletti X, et al. Role of chemotherapy for advanced/recurrent gastric cancer: an individualpatient-data meta-analysis. Eur J Cancer. 2013;49(7):1565-1577.

19. Chen D, Lin X, Zhang C, et al. Dual PI3K/mTOR inhibitor BEZ235 as a promising therapeutic strategy against paclitaxel-resistant gastric cancer via targeting PI3K/Akt/mTOR pathway. Cell Death Dis. 2018;9:123.

20. Kong F, Sun T, Kong X, Xie D, Li Z, Xie K. Krüppel-like factor 4 suppresses serine/threonine kinase 33 activation and metastasis of gastric cancer through reversing epithelial-mesenchymal transition. Clin Cancer Res. 2018;24(10):2440-2451.

21. Weng J, Xiao J, Mi Y, et al. PCDHGA9 acts as a tumor suppressor to induce tumor cell apoptosis and autophagy and inhibit the EMT process in human gastric cancer. Cell Death Dis. 2018;9(2):27.

22. Nappi TC, Salerno P, Zitzelsberger H, Carlomagno F, Salvatore G, Santoro M. Identification of polo-like kinase 1 as a potential therapeutic target in anaplastic thyroid carcinoma. Cancer Res. 2009;69(5):1916-1923.

23. Kanaji S, Saito H, Tsujitani S, et al. Expression of polo-like kinase 1 (PLK1) protein predicts the survival of patients with gastric carcinoma. Oncology. 2006;70(2):126-133.

24. Weichert W, Schmidt M, Gekeler V, et al. Polo-like kinase 1 is overexpressed in prostate cancer and linked to higher tumor grades. Prostate. 2004;60(3):240-245.

25. Cai XP, Chen LD, Song HB, Zhang CX, Yuan ZW, Xiang ZX. PLK1 promotes epithelial-mesenchymal transition and metastasis of gastric carcinoma cells. Am J Transl Res. 2016;8(10):4172-4183.
OncoTargets and Therapy

\section{Publish your work in this journal}

OncoTargets and Therapy is an international, peer-reviewed, open access journal focusing on the pathological basis of all cancers, potential targets for therapy and treatment protocols employed to improve the management of cancer patients. The journal also focuses on the impact of management programs and new therapeutic agents and protocols on

\section{Dovepress}

patient perspectives such as quality of life, adherence and satisfaction. The manuscript management system is completely online and includes a very quick and fair peer-review system, which is all easy to use. Visit http://www.dovepress.com/testimonials.php to read real quotes from published authors. 